\title{
Sentinel lymph node biopsy in cervical cancer: A meta-analysis
}

\author{
YIBO WU* ${ }^{*}$ ZEMING LI* , HAIYAN WU and JINJIN YU \\ Department of Gynecology and Obstetrics, Wuxi Fourth People's Hospital, \\ Jiangnan University, Wuxi, Jiangsu 214062, P.R. China
}

Received March 22, 2013; Accepted July 24, 2013

DOI: $10.3892 /$ mco.2013.168

\begin{abstract}
Sentinel lymph node biopsy (SLNB) is used to determine the pathological status of the first lymph node receiving lymphatic drainage from the primary tumor. The diagnostic value of SLNB for the assessment of the nodal status in patients with cervical cancer has not been determined. Therefore, this meta-analysis was conducted to assess the diagnostic value of SLNB in cervical cancer. A search for related literature was performed using PubMed. Data were analyzed using a random effects model and a subgroup analysis was used to assess the diagnostic value of different practices during the process. A total of 17 studies involving a total of 1,112 patients were included in the analysis. The pooled sentinel lymph node (SLN) detection rate, sensitivity and negative predictive values were $92.2 \%$ [95\% confidence interval (CI): 88.3-94.8\%], $88.8 \%$ (95\% CI: $85.1-91.7 \%$ ) and $95.0 \%$ (95\% CI: $92.8-96.6 \%$ ), respectively. The subgroup analysis revealed that laparoscopy, tracer combination and immunohistochemistry were associated with a higher SLN detection rate. In conclusion, the SLNB in cervical cancer appears to be of diagnostic value, particularly the procedure with the combination of laparoscopy, combined tracers and immunohistochemistry. However, further studies are required to establish the clinical value of SLNB in cervical cancer.
\end{abstract}

\section{Introduction}

The concept of the sentinel lymph node (SLN) refers to the first lymph node to receive lymphatic drainage from the primary tumor. Sentinel lymph node biopsy (SLNB) is used to determine the local and regional lymph node status of solid tumors. It was first described by Cabanas (1) for the management of penile cancer and has been widely used as an effective regional nodal staging procedure after a 1992 landmark study on melanoma

Correspondence to: Dr Yibo Wu, Department of Gynecology and Obstetrics, Wuxi Fourth People's Hospital, Jiangnan University, 200 Huihe Road, Binhu, Wuxi, Jiangsu 214062, P.R. China

E-mail:moliaty@yahoo.com.cn

*Contributed equally

Key words: cervical cancer, sentinel lymph node, detection rate, meta-analysis patients (2). It is currently used in several solid tumors, including breast cancer and melanoma $(3,4)$. The application and validity of SLNB in cervical cancer should be practiced with caution, since the lymphatic drainage of the cervix is significantly more complicated, due to its midline position. Although a number of feasibility studies for SLNB in cervical cancer have been conducted, SLNB does not appear to be suitable for clinical application, due to the wide range of reported detection rates, from 55.5 (5) to $100 \%$ (6). A high detection rate may render SLBN in cervical cancer feasible in clinical practice, which may decrease the complications, such as prolonged operation time, blood loss, lymphocyst and lymphedema, experienced by patients undergoing lymph node dissection (7). The pelvic nodal involvement rate in early-stage cervical cancer cases eligible for surgery was reported to be $0-4.8 \%$ in stage IA, $17 \%$ in stage IB and $12-27 \%$ in stage IIA disease $(8,9)$, suggesting that lymph node dissection may not be beneficial in $>90 \%$ of stage IA cases. Therefore, a reliable SLBN is crucial.

There is currently no clear assessment of SLNB diagnostic performance. Therefore, a meta-analysis of the published studies was conducted, with the aim to provide a comprehensive and up-to-date overview of the feasibility and diagnostic value of SLNB in cervical cancer.

\section{Materials and methods}

Search strategy. A comprehensive, systematic search for published studies was performed using the search terms 'cervical cancer', 'sentinel lymph node', 'sensitivity' and 'negative predictive value' in the PubMed database, with a time cutoff of September, 2012. The selected articles were limited to the English language. Reviews, comments, letters, conference abstracts and case reports were excluded from this analysis. Publications with a sample size of $<10$ were also excluded, since they were considered as case reports (10). The SLNB appears to be a better testing method compared to positron emission tomography, magnetic resonance imaging and computed tomography (11). Furthermore, surgical resection is the preferred therapeutic method for early cervical cancer. Therefore, we analyzed the studies in which sentinel lymph nodes (SLNs) were detected by the blue dye technique and/or by the use of a radiotracer intraoperatively.

Data extraction. Two independent investigators carefully extracted data from the selected articles using predefined tables, including first author, year of publication, sample size, route of 
Table I. Characteristics of the 17 studies included in the meta-analysis.

\begin{tabular}{|c|c|c|c|c|c|c|}
\hline First author & Year & $\begin{array}{l}\text { Sample } \\
\text { size }\end{array}$ & Route of surgery & $\begin{array}{l}\text { Detection } \\
\text { method }\end{array}$ & $\begin{array}{c}\text { Pathological } \\
\text { assessment }\end{array}$ & Refs. \\
\hline Malur & 2001 & 50 & Laparoscopy/laparotomy & $\mathrm{D}+\mathrm{I}$ & $\mathrm{HE}$ & (5) \\
\hline Levenback & 2002 & 39 & Laparotomy & $\mathrm{D}+\mathrm{I}$ & $\mathrm{HE}$ & (6) \\
\hline Rhim & 2002 & 26 & Laparotomy & $\mathrm{D}+\mathrm{I}$ & $\mathrm{HE}+\mathrm{IHC}$ & $(14)$ \\
\hline Lambaudie & 2003 & 12 & Laparoscopy & $\mathrm{D}+\mathrm{I}$ & $\mathrm{HE}+\mathrm{IHC}$ & $(15)$ \\
\hline Plante & 2003 & 70 & Laparoscopy & $\mathrm{D}+\mathrm{I}$ & $\mathrm{HE}+\mathrm{IHC}$ & $(16)$ \\
\hline Niikura & 2004 & 20 & Laparotomy & $\mathrm{D}+\mathrm{I}$ & $\mathrm{HE}+\mathrm{IHC}$ & $(17)$ \\
\hline Roca & 2005 & 40 & Laparoscopy/laparotomy & $\mathrm{D}+\mathrm{I}$ & $\mathrm{HE}+\mathrm{IHC}$ & $(18)$ \\
\hline Silva & 2005 & 56 & Laparotomy & Isotope & $\mathrm{HE}+\mathrm{IHC}$ & $(19)$ \\
\hline Wydra & 2006 & 100 & Laparotomy & $\mathrm{D}+\mathrm{I}$ & $\mathrm{HE}+\mathrm{IHC}$ & $(20)$ \\
\hline Zhang & 2006 & 27 & ND & $\mathrm{D}+\mathrm{I}$ & $\mathrm{HE}$ & $(21)$ \\
\hline Schwendinger & 2006 & 47 & Laparotomy & Dye & $\mathrm{HE}+\mathrm{IHC}$ & $(22)$ \\
\hline Kara & 2008 & 32 & Laparotomy & Isotope & $\mathrm{HE}+\mathrm{IHC}$ & $(23)$ \\
\hline Pazin & 2009 & 50 & ND & Dye & ND & $(24)$ \\
\hline Darlin & 2010 & 105 & Laparoscopy/laparotomy & Isotope & $\mathrm{HE}+\mathrm{IHC}$ & $(25)$ \\
\hline Ogawa & 2010 & 82 & ND & Isotope & $\mathrm{HE}$ & $(26)$ \\
\hline Lecuru & 2011 & 145 & Laparoscopy & $\mathrm{D}+\mathrm{I}$ & $\mathrm{HE}+\mathrm{IHC}$ & $(27)$ \\
\hline Roy & 2011 & 211 & Laparoscopy & $\mathrm{D}+\mathrm{I}$ & $\mathrm{HE}+\mathrm{IHC}$ & $(28)$ \\
\hline
\end{tabular}

$\mathrm{D}+\mathrm{I}$, dye + isotope; HE, hematoxylin-eosin staining; IHC, immunohistochemistry; ND, not described.

surgery, detection method, type of pathological assessment and diagnostic results (detection rate, mean SLN number, bilateral detection rate, sensitivity and negative predictive value).

Statistical analysis. The detection rate, sensitivity and negative predictive values were pooled with the random effects model of DerSimonian and Laird (13), using MetaAnalyst Beta 3.13 software (Tufts Medical Center, Boston, MA, USA) (12). The potential heterogeneity among the studies was assessed using the Q-statistic and $\mathrm{P}<0.1$ was considered to indicate a statistically significant difference. If heterogeneity was present, a subgroup analysis was used for further assessment.

\section{Results}

Study selection and description. A total of 38 studies were identified with the established search strategy. Studies that were clearly not eligible, as indicated by the information provided in the abstract, were excluded. For the remaining studies, the full text was read. Finally, a total of 17 studies were included in the analysis, involving a total of 1,112 patients $(5,6,14-28)$. The study selection process is summarized in Fig. 1. The median number of included patients per study was 50 (range, 12-211). The detailed characteristics of the 17 eligible studies are summarized in Table I and the diagnostic performance of SLNB is summarized in Table II.

Analysis of the 17 studies. The diagnostic value of SLNB was affected by several factors and significant heterogeneity was identified (Table I). Therefore, data were pooled using a random effects model.

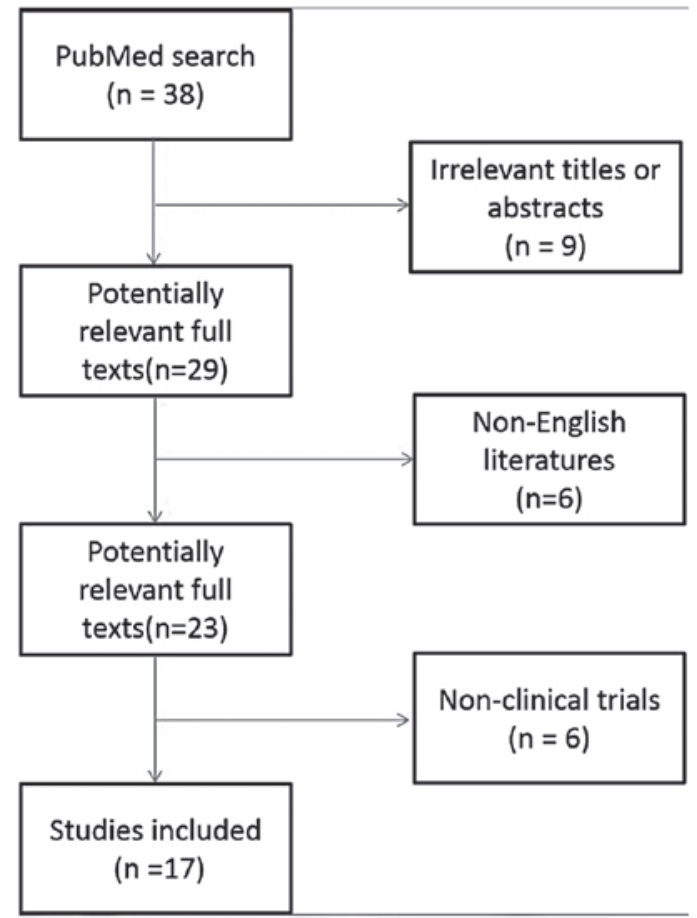

Figure 1. Flow chart of study selection process for the meta-analysis.

The pooled detection rate of SLN was $92.2 \%$ (95\% CI: 88.3-94.8\%; Fig. 2), whereas the pooled sensitivity and negative predictive values were 88.8 and $95.0 \%$, respectively (Fig. 3A and B). 
Table II. Diagnostic performance of the 17 studies included in the meta-analysis.

\begin{tabular}{lcccccccc}
\hline First author & Year & $\begin{array}{c}\text { Sample } \\
\text { size }\end{array}$ & $\begin{array}{c}\text { Detection } \\
\text { rate }(\%)\end{array}$ & $\begin{array}{c}\text { Bilateral } \\
\text { detection } \\
\text { rate }(\%)\end{array}$ & $\begin{array}{c}\text { Mean no. } \\
\text { of SLNs (\%) }\end{array}$ & Sensitivity & $\begin{array}{c}\text { Negative } \\
\text { predictive } \\
\text { value (\%) }\end{array}$ & Refs. \\
\hline Malur & 2001 & 50 & 78 & NA & 2 & 83.3 & 97 & $(5)$ \\
Levenback & 2002 & 39 & 100 & 72 & 4 & 87.5 & 97 & $(6)$ \\
Rhim & 2002 & 26 & 100 & NA & 2 & 80 & 95.2 & $(14)$ \\
Lambaudie & 2003 & 12 & 92 & 83 & 3.1 & 66.7 & 90 & $(15)$ \\
Plante & 2003 & 70 & 87 & 60 & 1.9 & 100 & 100 & $(16)$ \\
Niikura & 2004 & 20 & 90 & 75 & 2.3 & 100 & 100 & $(17)$ \\
Roca & 2005 & 40 & 100 & NA & 2.5 & 100 & 100 & $(18)$ \\
Silva & 2005 & 56 & 93 & 41 & 2.3 & 82.3 & 92.1 & $(19)$ \\
Wydra & 2006 & 100 & 84 & 66 & 1.8 & 86.4 & 95.5 & $(20)$ \\
Zhang & 2006 & 27 & 100 & 74 & 2.6 & 85.7 & 95.2 & $(21)$ \\
Schwendinger & 2006 & 47 & 83 & NA & 2 & 90 & 97 & $(22)$ \\
Kara & 2008 & 32 & 100 & 50 & 2.1 & 100 & 100 & $(23)$ \\
Pazin & 2009 & 50 & 92 & 38 & 2.6 & 85 & 89.6 & $(24)$ \\
Darlin & 2010 & 105 & 90 & 59 & 1 & 94 & 99 & $(25)$ \\
Ogawa & 2010 & 82 & 88 & 64 & 2.2 & 100 & 100 & $(26)$ \\
Lecuru & 2011 & 145 & 98 & 76 & 3 & 92 & 98.2 & $(27)$ \\
Roy & 2011 & 211 & 99 & 86 & NA & 90.6 & $94.2^{\text {a }}$ & $(28)$ \\
\hline
\end{tabular}

${ }^{a}$ Calculated from 181 patients in whom bilateral sentinel lymph nodes were identified. SLN, sentinel lymph node; NA, not available.

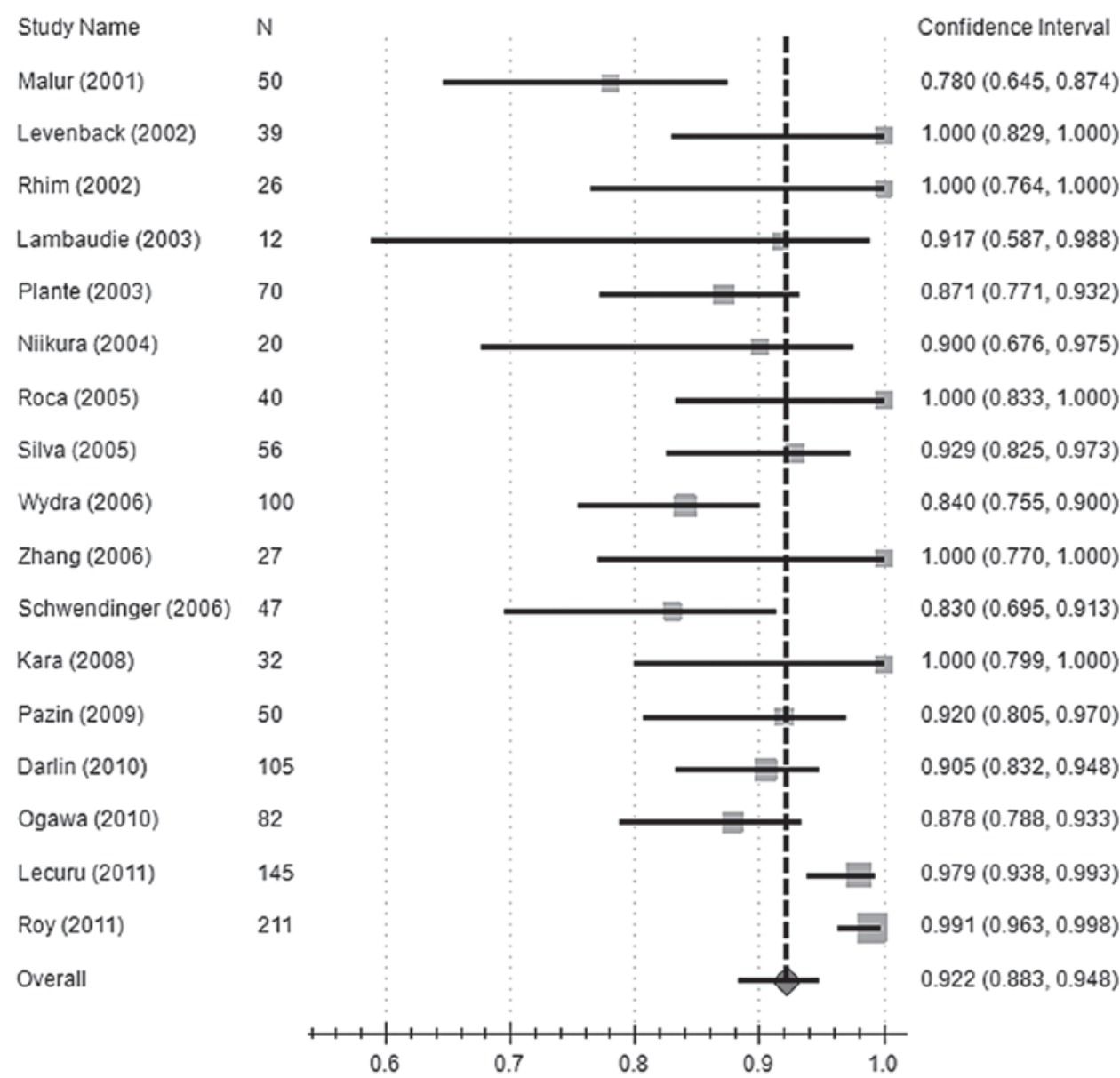

Figure 2. Forest plot showing the detection rate of sentinel lymph node biopsy in the 17 studies. 


\begin{tabular}{ll} 
Study Name & $\mathrm{N}$ \\
Malur (2001) & 50 \\
Levenback (2002) & 39 \\
Rhim (2002) & 26 \\
Lambaudie (2003) & 12 \\
Plante (2003) & 70 \\
Niikura (2004) & 20 \\
Roca (2005) & 40 \\
Silva (2005) & 56 \\
Wydra (2006) & 100 \\
Zhang (2006) & 27 \\
Schwendinger (2006) & 47 \\
Kara (2008) & 32 \\
Pazin (2009) & 50 \\
Darlin (2010) & 105 \\
Ogawa (2010) & 82 \\
Lecuru (2011) & 145 \\
Roy (2011) & 211 \\
Overall & \\
\hline
\end{tabular}

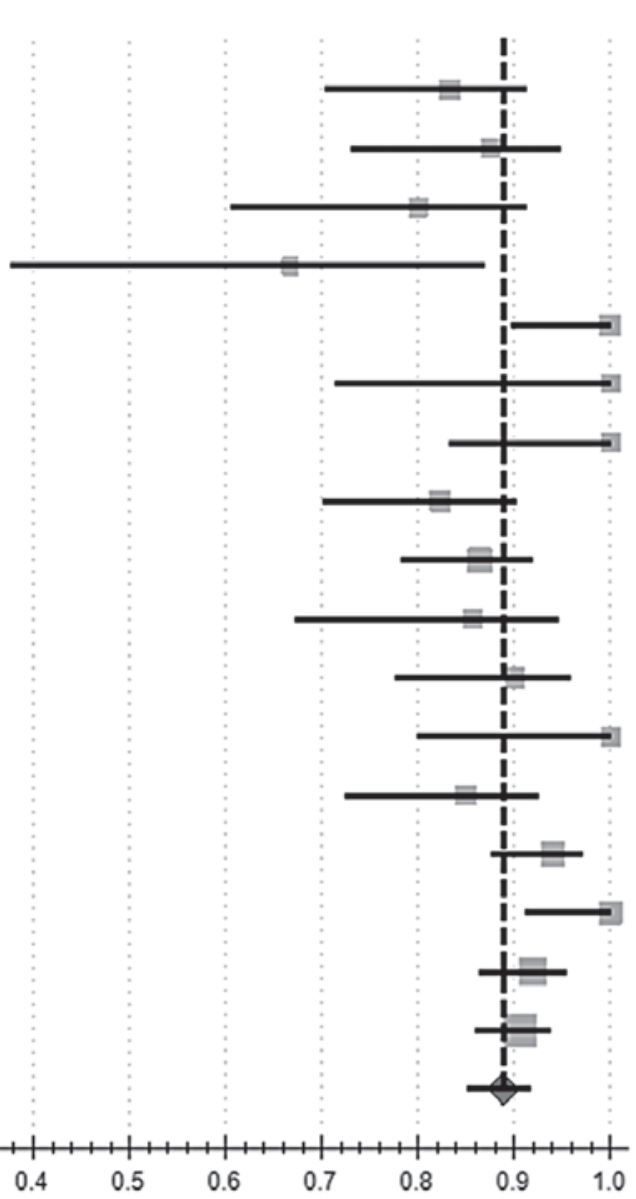

Confidence Interval $0.833(0.703,0.913)$ $0.875(0.730,0.948)$ $0.800(0.605,0.913)$ $0.667(0.376,0.869)$ $1.000(0.897,1.000)$ $1.000(0.713,1.000)$ $1.000(0.833,1.000)$ $0.823(0.701,0.902)$ $0.864(0.782,0.918)$ $0.857(0.671,0.946)$ $0.900(0.776,0.959)$ $1.000(0.799,1.000)$ $0.850(0.723,0.925)$ $0.940(0.875,0.972)$ $1.000(0.911,1.000)$ $0.920(0.863,0.954)$ $0.906(0.859,0.939)$ $0.888(0.851,0.917)$ B Study Name N

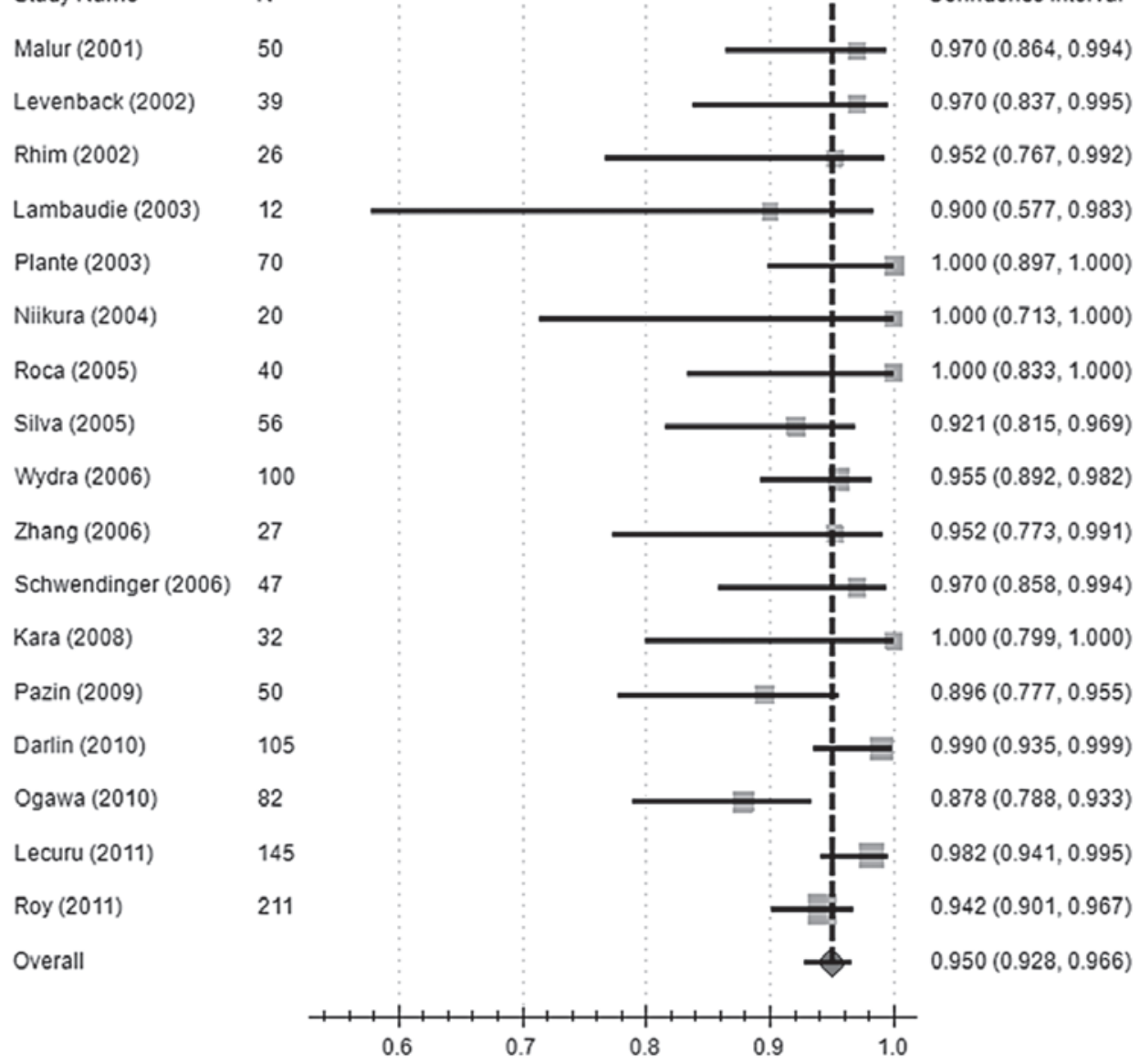

Figure 3. Forest plot showing (A) the sensitivity and (B) the negative predictive value of sentinel lymph node biopsy in the 17 studies. 
Table III. Meta-analysis results of the 17 studies.

\begin{tabular}{lccc}
\hline Diagnostic parameters & Detection rate \% (95\% CI) & Sensitivity \% (95\% CI) & Negative predictive value\% (95\% CI) \\
\hline Overall (17 studies) & $92.2(88.3-94.8)$ & $88.8(85.1-91.7)$ & $95.0(92.8-96.6)$ \\
Laparoscopy (4 studies) & $96.1(85.5-99.0)$ & $89.8(79.5-95.2)$ & $96.2(90.9-98.5)$ \\
Laparotomy (7 studies) & $90.2(83.1-94.5)$ & $86.3(81.4-90.1)$ & $95.3(92.2-97.2)$ \\
Dye (2 studies) & $87.5(75.3-94.1)$ & $87.2(78.9-92.6)$ & $93.2(79.9-97.9)$ \\
Isotope (4 studies) & $90.3(86.0-93.4)$ & $94.4(82.6-98.4)$ & $94.5(85.6-98.1)$ \\
D+I (11 studies) & $94.3(88.5-97.2)$ & $88.0(83.2-91.6)$ & $95.7(93.8-97.0)$ \\
HE (4 studies) & $89.4(75.8-95.8)$ & $88.0(77.3-94.1)$ & $93.7(86.1-97.3)$ \\
HE+IHC (12 studies) & $93.1(88.6-96.0)$ & $89.6(85.0-92.9)$ & $95.7(93.7-97.1)$ \\
\hline
\end{tabular}

CI, confidence interval; D+I, dye + isotope; HE, hematoxylin-eosin staining; IHC, immunohistochemistry.

Subgroup analysis of three factors. The Q-statistic P-values of the heterogeneity test were $<0.1$. We performed subgroup analyses according to the route of surgery (laparoscopy or laparotomy), the detection method (dye, isotope or a combination of the two), pathological assessment type [hematoxylin-eosin staining (HE) or HE + immunohistochemistry (IHC)]. The results of the subgroup analyses are presented in Table III.

When considering the route of surgery, the pooled SLN detection rate in the laparoscopy subgroup (4 studies) was $96.1 \%$, compared to $90.2 \%$ in the laparotomy subgroup (7 studies). The sensitivity and negative predictive values of the two subgroups were 89.8 vs. $86.3 \%$ and 96.2 vs. $95.3 \%$, respectively.

When considering the detection method, the pooled SLN detection rate in dye subgroup (2 studies), the isotope subgroup (4 studies) and the combination of the two subgroup (11 studies) was $87.5,90.3$ and $94.3 \%$, respectively. The pooled negative predictive value of the combination subgroup was higher compared to that of the dye and isotope subgroups.

When considering the pathological assessment type, the pooled detection rates in the HE+IHC subgroup (12 studies) was $93.1 \%$ and in the HE subgroup $89.4 \%$ (4 studies).

\section{Discussion}

In the present meta-analysis we pooled the detection rate, sensitivity and negative predictive values with data extracted from 17 studies. The overall SLN detection rate was $92.2 \%$, which was satisfactory in the SLNB of cervical cancer, whereas the high pooled sensitivity $(88.8 \%)$ and negative predictive values $(95.0 \%)$ also indicated that this procedure is feasible.

However, the diagnostic parameters were affected by several factors, such as the route of surgery, the detection method, the pathological assessment type and other predictable/unpredictable factors. Subgroup analyses were performed for the three factors mentioned above.

First, in the subgroup analysis according to the route of surgery, we observed that the detection rate of laparoscopy was superior to that of laparotomy (96.1 vs. 90.2\%). Furthermore, laparoscopy exhibited a higher sensitivity compared to laparotomy ( 89.8 vs. $86.3 \%$ ), with wide visual fields and minimal incisions. Therefore, the technologically improved laparo- scopic equipment is recommended for the surgical treatment of cervical cancer.

Second, the SLN detection rate in cervical cancer with the combination of dye and isotope $(94.3 \%)$ was higher compared to that of dye $(87.5 \%)$ or isotope $(90.3 \%)$ alone. In addition, the pooled negative predictive value exhibited the same trend. Van de Lande et al (29) also reported that the combination of a radionuclide with a blue dye was the optimal method of SLN detection in a systematic review that mainly compared the three methods.

Finally, when the pathological assessment with HE + IHC was used to determine the lymph node status, higher detection and sensitivity rates were achieved compared to the $\mathrm{HE}$ group (93.1 vs. $89.4 \%$ and 89.6 vs. $88.0 \%$, respectively). IHC may accurately determine the lymph node status, since it is able to detect micrometastases, compared to HE alone (30). Therefore, under the appropriate conditions, IHC is recommended in SLNB.

In this meta-analysis, the predetermined search strategy described above was used for the selection of studies from the available literature. Several studies on SLNB in cervical cancer were identified; however, the data used by each study to describe the performance characteristics were inconsistent, due to the different objectives of the studies. Several studies reported data that were not sufficient to calculate sensitivity or negative predictive values, leading to the exclusion of those studies.

Furthermore, in view of the limited time and capacity, newly published literature was not included in our study.

In conclusion, we analyzed the performance characteristics of SLNB in cervical cancer using data from 17 studies, including a total of 1,112 patients. Although SLNB appears to exhibit a satisfactory diagnostic performance in the meta-analysis, further studies are required to determine the true performance of the clinical application of SLNB in cervical cancer.

\section{References}

1. Cabanas RM: An approach for the treatment of penile carcinoma. Cancer 39: 456-466, 1977.

2. Morton DL, Wen DR, Wong JH, Economou JS, Cagle LA, Storm FK, et al: Technical details of intraoperative lymphatic mapping for early stage melanoma. Arch Surg 127: 392-399, 1992. 
3. Morrow M, Rademaker AW, Bethke KP, Talamonti MS, Dawes LG, Clauson J and Hansen N: Learning sentinel node biopsy: results of a prospective randomized trial of two techniques. Surgery 126: 714-720, 1999.

4. Lyman GH, Giuliano AE, Somerfield MR, et al; American Society of Clinical Oncology: American Society of Clinical Oncology guideline recommendations for sentinel lymph node biopsy in early-stage breast cancer. J Clin Oncol 23: 7703-7720, 2005.

5. Malur S, Krause N, Kohler C and Schneider A: Sentinel lymph node detection in patients with cervical cancer. Gynecol Oncol 80: 254-257, 2001.

6. Levenback C, Coleman RL, Burke TW, Lin WM, Erdman W, Deavers $\mathrm{M}$ and Delpassand ES: Lymphatic mapping and sentine node identification in patients with cervix cancer undergoing radical hysterectomy and pelvic lymphadenectomy. J Clin Oncol 20: 688-693, 2002.

7. Achouri A, Huchon C, Bats AS, Bensaid C, Nos C and Lecuru F: Complications of lymphadenectomy for gynecologic cancer. Eur J Surg Oncol 39: 81-86, 2013

8. Ayhan A, Celik H, Dursun P, Gultekin M and Yuce K: Prognostic and therapeutic importance of lymphadenectomy in gynecological cancers. Eur J Gynaecol Oncol 25: 279-286, 2004.

9. Hauspy J, Beiner M, Harley I, Ehrlich L, Rasty G and Covens A: Sentinel lymph node in vulvar cancer. Cancer 110: 1015-1023, 2007.

10. Kang S, Yoo HJ, Hwang JH, Lim MC, Seo SS and Park SY Sentinel lymph node biopsy in endometrial cancer: meta-analysis of 26 studies. Gynecol Oncol 123: 522-527, 2011

11. Selman TJ, Mann C, Zamora J, Appleyard TL and Khan K: Diagnostic accuracy of tests for lymph node status in primary cervical cancer: a systematic review and meta-analysis. CMAJ 178: 855-862, 2008

12. Wallace BC, Schmid CH, Lau J and Trikalinos TA: Meta-Analyst: software for meta-analysis of binary, continuous and diagnostic data. BMC Med Res Methodol 9: 80, 2009.

13. DerSimonian R and Laird N: Meta-analysis in clinical trials Control Clin Trials 7: 177-188, 1986.

14. Rhim CC, Park JS, Bae SN and Namkoong SE: Sentinel node biopsy as an indicator for pelvic nodes dissection in early stage cervical cancer. J Korean Med Sci 17: 507-511, 2002.

15. Lambaudie E, Collinet P, Narducci F, Sonoda Y, Papageorgiou T, Carpentier P, Leblanc E and Querleu D: Laparoscopic identification of sentinel lymph nodes in early stage cervical cancer: prospective study using a combination of patent blue dye injection and technetium radiocolloid injection. Gynecol Oncol 89: 84-87, 2003.

16. Plante M, Renaud MC, Tetu B, Harel F and Roy M: Laparoscopic sentinel node mapping in early-stage cervical cancer. Gynecol Oncol 91: 494-503,2003
17. Niikura H, Okamura C, Akahira J, Takano T, Ito K, Okamura K and Yaegashi N: Sentinel lymph node detection in early cervical cancer with combination ${ }^{99 \mathrm{~m}} \mathrm{Tc}$ phytate and patent blue. Gynecol Oncol 94: 528-552, 2004.

18. Roca I, Caresia AP, Gil-Moreno A, et al: Usefulness of sentinel lymph node detection in early stages of cervical cancer. Eur J Nucl Med Mol Imaging 32: 1210-1216, 2005.

19. Silva LB, Silva-Filho AL, Traiman P, et al: Sentinel node detection in cervical cancer with ${ }^{99} \mathrm{~m}$ Tc-phytate. Gynecol Oncol 97: 588-595, 2005.

20. Wydra D, Sawicki S, Wojtylak S, Bandurski T and Emerich J: Sentinel node identification in cervical cancer patients undergoing transperitoneal radical hysterectomy: a study of 100 cases. Int J Gynecol Cancer 16: 649-654, 2006.

21. Zhang WJ, Zheng R, Wu LY, Li XG, Li B and Chen SZ: Clinical application of sentinel lymph node detection to early stage cervical cancer. Chin J Cancer 25: 224-228, 2006 (In Chinese).

22. Schwendinger V, Muller-Holzner E, Zeimet AG and Marth C: Sentinel node detection with the blue dye technique in early cervical cancer. Eur J Gynaecol Oncol 27: 359-362, 2006

23. Kara PP, Ayhan A, Caner B, Gultekin M, Ugur O, Bozkurt MF and Usubutun A: Sentinel lymph node detection in early stage cervical cancer: a prospective study comparing preoperative lymphoscintigraphy, intraoperative gamma probe, and blue dye. Ann Nucl Med 22: 487-494, 2008.

24. Pazin V, Dragojević S, Miković Z, et al: The value of sentinel lymphadenectomy in radical operative treatment of cervical cancer. Mil Med Pharm Rev 66: 539-543, 2009.

25. Darlin L, Persson J, Bossmar T, Lindahl B, Kannisto P, Måsbäck A and Borgfeldt $C$ : The sentinel node concept in early cervical cancer performs well in tumors smaller than $2 \mathrm{~cm}$. Gynecol Oncol 117: 266-269, 2010.

26. Ogawa S, Kobayashi H, Amada S, et al: Sentinel node detection with ${ }^{99 \mathrm{~m}} \mathrm{Tc}$ phytate alone is satisfactory for cervical cancer patients undergoing radical hysterectomy and pelvic lymphadenectomy. Int J Clin Oncol 15: 52-58, 2010.

27. Lécuru F, Mathevet P, Querleu D, et al: Bilateral negative sentinel nodes accurately predict absence of lymph node metastasis in early cervical cancer: results of the SENTICOL study. J Clin Oncol 29: 1686-1691, 2011.

28. Roy M, Bouchard-Fortier G, Popa I, Grégoire J, Renaud MC, Têtu B and Plante M: Value of sentinel node mapping in cancer of the cervix. Gynecol Oncol 122: 269-274, 2011.

29. Van de Lande J, Torrenga B, Raijmakers PG, Hoekstra OS, van Baal MW, Brölmann HA and Verheijen RH: Sentinel lymph node detection in early stage uterine cervix carcinoma: a systematic review. Gynecol Oncol 106: 604-613, 2007.

30. Van Trappen PO and Pepper MS: Lymphatic dissemination of tumour cells and the formation of micrometastases. Lancet Oncol 3: 44-52, 2002. 